\title{
Criminologie
}

\section{L’analyse stratégique et quelques développements récents en criminologie}

\section{Maurice Cusson}

Volume 19, numéro 1, 1986

Politiques et pratiques pénales. 25 ans de réflexion et d'action

URI : https://id.erudit.org/iderudit/017226ar

DOI : https://doi.org/10.7202/017226ar

Aller au sommaire du numéro

\section{Éditeur(s)}

Les Presses de l'Université de Montréal

ISSN

0316-0041 (imprimé)

1492-1367 (numérique)

Découvrir la revue

Citer cet article

Cusson, M. (1986). L'analyse stratégique et quelques développements récents en criminologie. Criminologie, 19(1), 53-72. https://doi.org/10.7202/017226ar

\section{Résumé de l'article}

Strategic analysis views crime as a confrontation and as a mean to an end. It is characterised by : 1) it concentrates on crime; 2 ) it takes cognizance of the circumstances under which the crime is committed; 3) it presents the crime as a decision influenced by its anticipated results. Felson's routine activity approach, which is similar to strategic analysis, is presented in this article. Other recent developments in criminology have made it possible to present several assertions with a view to explaining certain aspects of theft, in particular, the choice of target. These assertions are : 1) thefts vary according to the opportunities offered potential thieves; 2) opportunity is defined as the contact between a potential criminal and a suitable target; 3 ) the number of contacts between potential criminals and suitable targets varies directly with the number of targets and their accessibility; 4) the suitability of targets varies in direct proportion to their value and vulnerability. It varies in inverse proportion to their inertia. 


\section{L'ANALYSE STRATÉGIQUE ET QUELQUES DÉVELOPPEMENTS RÉCENTS EN CRIMINOLOGIE}

Maurice Cusson*

Strategic analysis views crime as a confrontation and as a mean to an end. It is characterised by : 1) it concentrates on crime; 2) it takes cognizance of the circumstances under which the crime is committed; 3) it presents the crime as a decision influenced by its anticipated results. Felson's routine activity approach, which is similar to strategic analysis, is presented in this article. Other recent developments in criminology have made it possible to present several assertions with a view to explaining certain aspects of theft, in particular, the choice of target. These assertions are : 1) thefts vary according to the opportunities offered potential thieves; 2) opportunity is defined as the contact between a potential criminal and a suitable target; 3) the number of contacts between potential criminals and suitable targets varies directly with the number of targets and their accessibility; 4) the suitability of targets varies in direct proportion to their value and vulnerability, It varies in inverse proportion to their inertia.

\section{INTRODUCTION}

Dans son sens restreint, la stratégie désigne la conduite des opérations militaires; dans son sens général, elle est l'«art de coordonner des actions et de manœuvrer pour atteindre un but» (Larousse). L'analyse stratégique en criminologie emprunte à ces deux sens. L'idée de conflit y est : le délit est vu comme l'élément central d'un affrontement entre le délinquant et sa victime; entre le délinquant et les forces de l'ordre. Plus important encore, elle évoque aussi l'idée d'une action en vue d'une fin: le crime apparaît comme un moyen utilisé par le délinquant pour réaliser ses fins.

Si on est fondé de considérer le délit comme un moyen en vue d'une fin, les caractéristiques de la cible visée par le délinquant devraient l'influencer. C'est effectivement ce que tendent à démontrer une série de travaux récents en criminologie. Or, ces contributions n'étaient pas prises en compte ni dans Délinquants

\footnotetext{
- Professeur titulaire, École de criminologie, Université de Montréal.
} 
pourquoi? (Cusson, 1981) ni dans le Contrôle social du crime (Cusson, 1983). Cet article vise à combler cette lacune. Par la même occasion, je présenterai un certain nombre de travaux qui n'avaient pas été évoqués dans les ouvrages mentionnés même s'ils adoptaient un point de vue très semblable à celui de l'analyse stratégique. C'est ainsi que seront résumés des travaux de recherches qui montrent que l'évolution de la criminalité est reliée aux changements dans les habitudes de vie des gens (Cohen et Felson, 1979; Felson et Cohen, 1980; Cohen, Felson et Land, 1980; Felson, 1983). Par la suite, je proposerai un modèle théorique centré sur la notion d'opportunité qui tente d'intégrer plusieurs observations faites au cours de recherches sur le processus de prises de décisions des délinquants, sur la prévention «situationnelle», sur le choix de la cible et sur les facteurs associés à la victimisation (Brantingham et Brantingham, 1978, 1980; Hindelang Gottfredson et Garofalo, 1978; Clarke, 1980; Van Dijk ct Steinmetz, 1980; Clarke et Mayhew, 1980; Clarke, 1983; Clarke et Cornish, 1985).

Malgré le caractère disparate de cette production et malgré l'absence de concertation entre ces chercheurs, il s'en dégage une remarquable communauté de perspective. Ces auteurs adoptent des points de vue semblables et leurs analyses convergent vers des conclusions complémentaires. Cette communauté de perspective se manifeste sur trois points.

1. L'attention se porte sur le crime et non plus sur le criminel, sur l'acte du délinquant plutôt que sur ses prédispositions, sur ses décisions plutôt que sur ses pulsions. Depuis le XIX ${ }^{e}$ siècle, des criminologues ont eu tendance à raisonner en termes de penchant au crime. La question essentielle était : "Comment deviennent-ils délinquants?» On voulait rendre compte du phénomène criminel par des caractéristiques installées à demeure chez certains individus : traits de caractère, mode de fonctionnement, normes sousculturelles intériorisées, identité négative façonnée par l'étiquetage... Toute explication passait obligatoirement par un individu doué d'une inclinaison au crime. La légitimité de cette démarche n'est pas en cause. Il est en effet difficile de nier qu'il y ait des individus plus portés au crime que d'autres. Il s'agit d'un acquis qu'on aurait mauvaise grâce à contester. Il n'en reste pas moins possible de partir de cet acquis et de se demander : dans quelles circonstances les tendances criminelles d'un individu se traduisent- 
elles en actes? Le penchant au crime est une condition nécessaire, elle n'est pas suffisante. Ceci nous conduit à un deuxième point de convergence.

2. On tient compte des circonstances dans lesquelles se produit un délit et qui le rendent possible. Pour réaliser son vol, le voleur doit entrer en contact avec une cible susceptible de le satisfaire. Dans certains cas, il ne trouvera rien malgré toutes ses démarches. Et alors, il ne commettra pas de vol et devra revenir chez lui bredouille comme le chasseur qui n'a pas tué son gibier. Ainsi donc, l'occasion, l'opportunité a un rôle déterminant à jouer dans le crime. Le voleur qui a la chance de tomber sur un grand nombre de cibles importantes commettra plus de vols que son collègue qui n'a pas cette chance.

3. Le délit est vu comme une décision influencée par ses résultats anticipés et non comme l'effet d'une cause quelconque. Ceci nous conduit à concevoir le délinquant comme un être actif qui profite des opportunités qui s'offrent à lui pour maximiser ses gains. Le crime apparaît alors comme le fruit d'une démarche rationnelle. Les limites de cette rationalité sont également reconnues. Le délinquant fait des erreurs de jugement, il raisonne à très court terme, il perçoit sélectivement l'information disponible et il l'analyse sommairement (cf. Clarke et Cornish, 1985). Malgré tout, le postulat de la rationalité s'avère utile. On comprend mieux la délinquance quand on présuppose que le délinquant fait une sommaire estimation des avantages et des inconvénients de son action quand il est sur le point d'agir.

L'analyse stratégique conduit les chercheurs à adopter un point de vue sensiblement différent de celui que l'on trouvait jusqu'à maintenant en criminologie. De ce fait, elle favorise l'émergence de questions également différentes, comme en fait foi l'énumération qui suit.

a) Quelles sont les étapes du processus de prise de décision qui conduit au crime?

b) Comment les délinquants préparent-ils leurs délits?

c) Comment les délinquants exécutent-ils leurs délits? (modus operandi, opérations, organisation...)

d) Quelles sont les fins poursuivies par les délinquants lors de l'exécution de leurs délits? 
e) Pourquoi les délinquants optent-ils pour tel type de délit, ou pour telle combinaison de délits?

f) Comment le délinquant choisit-il sa cible ou sa victime?

g) Jusqu'à quel point les opportunités influent-elles sur la décision de poser un acte criminel?

h) Les caractéristiques des cibles et des victimes influent-elles sur les décisions de commettre un crime?

i) Les risques de l'arrestation et la sévérité anticipée de la peine influent-ils sur la décision de commettre un crime?

j) Comment se prend la décision d'entreprendre une carrière criminelle?

k) Comment se prend la décision de poursuivre une carrière criminelle?

1) Comment se prend la décision d'abandonner une carrière criminelle?

$\mathrm{m})$ Les succès et les échecs dans le crime ont-ils une influence sur la décision de continuer ou d'abandonner une carrière criminelle?

n) Les sanctions pénales ont-elles une influence sur la décision de continuer ou d'abandonner une carrière criminelle?

\section{FELSON : «A ROUTINE ACTIVITY APPROACH»}

Les publications de Felson et de ses collègues offrent une bonne illustration de l'intérêt et de la fécondité de la démarche qui vient d'être présentée. Ces auteurs désignent leur théorie par les mots "routine activity", que l'on peut traduire par habitudes de vie ou encore par activités habituelles. Il s'agit de toutes activités qui se répètent assez fréquemment pour faire partie de la vie quotidienne des gens et qui servent à satisfaire leurs principaux besoins : nourriture, logement, loisirs, éducation... Elles se cristallisent dans une structure spacio-temporelle qui obéit aux contraintes de la vie professionnelle, familiale, scolaire et de loisirs des gens.

La thèse centrale de Felson est que la criminalité est façonnée par les activités habituelles. Selon la manière dont les gens organisent leur vie quotidienne, les opportunités qui s'offriront aux délinquants potentiels seront plus ou moins nombreuses; les cibles 
qui se présenteront à eux seront plus ou moins intéressantes et elles seront plus ou moins bien défendues.

La démonstration comporte deux aspects distincts mais étroitement indépendants. Premièrement, on nous propose un modèle théorique que l'on peut résumer en une phrase : la probabilité qu'un crime soit commis en un lieu et à un moment donné est fonction de la convergence spatio-temporelle de délinquants potentiels, de cibles intéressantes et de l'absence de gardien capable de les protéger (Felson et Cohen, 1980, p. 392). Deuxièmement, on nous montre que l'évolution de la criminalité entre 1950 et 1975 va de pair avec d'importants changements dans les habitudes de vie des Américains pendant cette période. Nous verrons maintenant ces deux éléments de la démonstration plus en détails.

1. Le modèle théorique est simple et d'un grand niveau de généralité. Il tend à répondre à la question : quelles sont les conditions nécessaires à la perpétration d'un crime «prédateur» (cambriolage, vol qualifié, agression, viol, homicide) ? La réponse : il faut que soient réunis au minimum trois éléments :

a) un délinquant potentiel c'est-à-dire un individu qui est porté au crime et qui en est capable;

b) une cible intéressante pour le délinquant;

c) l'absence de gardien capable de prévenir le crime.

Il suffit qu'une seule de ces conditions ne soit pas remplie pour que le crime n'ait pas lieu. (Cohen et Felson, 1979, p. 589). Inversement, tout ce qui contribue à faire converger à un moment et en un lieu donné ces trois éléments, contribue à la criminalité.

À quelles conditions une cible paraîtra-t-elle intéressante au délinquant potentiel? Quatre dimensions permettent de spécifier le concept. Premièrement, la Valeur. Il s'agit des attributs qui rendent un objet ou une personne désirable pour le délinquant : valeur monétaire (le cas échéant, la valeur de revente) valeur symbolique, l'attirance sexuelle, etc. Deuxièmement, la visibilité : tout ce qui facilite la découverte de la cible par le délinquant potentiel. Troisièmement, l'accessibilité : ce qui permet au délinquant d'entrer en contact avec la cible (le site et ce qui favorise l'entrée et la fuite). Quatrièmement, l'inertie : tout ce qui rend la cible plus ou moins difficile à déplacer. Ceci inclut : le poids, la dimension, la mobilité, les serrures, la capacité de résister... 
2. L'analyse de l'évolution de la criminalité aux États-Unis est à la fois une validation et une illustration du modèle théorique. Entre 1950 et 1975, la criminalité telle qu'elle est connue par les statistiques officielles, est en constante augmentation. Et pourtant, on est en période de croissance économique. De plus, entre 1960 et 1970 , le taux de chômage américain baisse considérablement, ce qui n'empêche pas la criminalité de croître (Cohen et Felson, 1979 , p. 604). Par contre, vers la même période se produit une importante transformation dans les habitudes de vie des gens : la proportion d'activités réalisées dans le cadre de la famille diminue constamment. Les femmes sont de plus en plus nombreuses à aller travailler hors de la maison. Les familles cessent de plus en plus d'être des unités d'activité économique et, de plus en plus, on doit quitter la maison pour aller gagner sa vie. Finalement, le nombre de foyers qui n'hébergent qu'un seul adulte (familles monoparentales et personnes vivant seules) est en progression constante. Or, cette évolution est nettement liée à l'augmentation de la criminalité. Cohen et Felson (1979, p. 600 et s.s.) créent un indice d'activités extra-familiales composé de deux éléments : 1) le nombre de femmes mariées qui travaillent et le nombre de familles monoparentales (ou de foyers formés d'un seul adulte). Ceci fait, ils mettent en relation l'évolution de cet indice de 1947 à 1974 avec l'évolution de divers taux de criminalité (homicide, viol, voies de fait, vol qualifié et cambriolage). Ils constatent alors qu'il y a une relation statistique positive et significative entre ces variables. Plus le taux d'activités extra-familiales augmente, plus la criminalité augmente. Felson et Cohen (1980) poursuivent en montrant que les taux de cambriolage aux États-Unis de 1950 à 1972 s'expliquent par trois variables: 1) le pourcentage d'individus âgés de 15 à 24 ans dans la population; 2) le poids des appareils de télévision et

3) le pourcentage de foyers n'hébergeant qu'un seul adulte. Cette dernière variable apporte la plus forte contribution à l'explication du cambriolage.

Comment se fait-il que la criminalité varie en raison inverse de la fréquencè des activités familiales? L'hypothèse de Felson repose sur l'idée déjà mentionnée que l'absence de gardien capable de protéger les biens est une condition de plusieurs crimes. Quand les gens quittent leur foyer (pour aller travailler, étudier ou s'amuser), des délinquants potentiels ont de meilleures chances d'entrer en contact avec des cibles non gardées. Il est relativement 
facile de se protéger et de protéger ses biens quand on reste à la maison. Mais si, pour vaquer à ses affaires, on doit constamment s'absenter de chez soi, on est moins bien placé pour surveiller ses biens et simultanément, on multiplie les occasions de contacts avec des inconnus qui peuvent être portés au crime. Le style de vie tel qu'il s'est développé depuis la guerre favorise cette plus grande vulnérabilité des biens et des personnes.

Cette analyse est corroborée par les faits suivants :

1. les sondages de victimisations ont établi que l'on est moins souvent victime de crime de violence à la maison où dans ses environs immédiats que partout ailleurs (Hindelang et al., 1977). Il semble donc que le foyer ait une fonction protectrice laquelle va en s'atténuant avec la diminution des activités familiales.

2. Les individus qui, à cause de leur âge ou de leur statut, passent plus de temps que les autres hors de la maison sont plus souvent victimisés. Les adolescents et les jeunes adultes sont plus souvent victimes de crimes que les personnes âgées. Les travailleurs le sont plus que les retraités. Les célibataires le sont plus que les gens mariés (Hindelang, Gottfredson et Garofalo, 1978).

3. Le taux de victimisation de ceux qui sont les seuls adultes à la maison est presque deux fois plus élevé que celui des gens qui vivent avec un ou d'autres adultes. Les gens qui vivent seuls sont plus portés à sortir de chez eux, laissant leurs biens sans surveillance et s'exposant eux-mêmes (Felson et Cohen, 1980, p. 398).

4. Entre 1960 et 1971 , le pourcentage de foyers dans lesquels il n'y avait aucune personne de 14 ans ou plus pendant le jour augmente de façon sensible. Durant cette même période, les cambriolages perpétrés pendant la journée augmentent beaucoup plus rapidement que les cambriolages faits la nuit ou que ceux qui visent les commerces (Cohen et Felson, 1979, pp. 598-600). On est donc fondé de penser que les cambriolages augmentent parce que les gens quittent leur foyer en le laissant sans surveillance.

5. Entre 1963 et 1975 , les homicides entre parents augmentent de $47 \%$ alors que les homicides commis par des criminels reconnus augmentent de $294 \%$ (Cohen et Felson, 1979, p. 600) : En d'autres termes, l'augmentation la plus forte concerne essentiellement des homicides dans lesquels des étrangers sont impliqués. Or c'est essentiellement durant des activités extra familiales que l'on risque d'être tué par un inconnu. 
L'absence de gardien capable d'empêcher le crime apparaît donc comme une dimension fort utile pour rendre compte de l'augmentation de la criminalité de l'après-guerre. Il n'en reste pas moins que le modèle théorique de base comportait deux autres dimensions : un délinquant potentiel et une cible intéressante. Quelquefois, Felson considère la variable «délinquant potentiel» comme une constante; d'autres fois, il l'introduit dans ses équations par le biais de la proportion de gens âgés de 14 à 25 ans dans la population. À la rubrique "cible intéressante», Cohen et Felson (1979, p. 599) proposent le raisonnement suivant. Pour qu'un objet intéresse un voleur, il ne doit pas être trop difficile à transporter, son inertie ne doit pas être trop grande. De ce fait, si le poids des appareils électroniques diminue, le nombre de cibles intéressantes augmentera. Nos auteurs consultent alors le catalogue de Sears : en 1960, la télévision la plus légère pesait 38 livres, en 1970 elle en pesait 15. Dans un dernier temps, on vérifie s'il y a une relation statistique entre le poids des téléviseurs et le cambriolage. De fait, la corrélation existe.

Commentaire. Le modèle qui vient d'être présenté satisfait à plusieurs des critères avec lesquels on juge de la validité d'une théorie. Il est cohérent et parcimonieux, nous permettant de rendre compte d'un grand nombre de faits avec un minimum de propositions. Son efficacité est particulièrement évidente au niveau macro-sociologique. Le modèle permet à Felson et à ses collègues de présenter l'explication la plus convaincante et la mieux documentée de l'augmentation de la criminalité de l'après-guerre. On nous démontre qu'elle découle de la dispersion des activités hors de la famille et, en définitive, de l'isolement des individus. Ce n'est pas la première fois qu'on nous parle de l'affaiblissement de la famille mais c'est la première fois qu'on nous montre que notre vulnérabilité augmente avec l'effritement de la famille. Celle-ci, comme les forteresses d'autrefois, contribuait à notre sécurité. Son affaiblissement nous rend plus vulnérable et augmente l'insécurité. Felson nous fait prendre conscience d'un changement social majeur : nous devenons des sociétés d'étrangers de moins en moins capables de se protéger contre le crime.

\section{OPPORTUNITÉS ET VOL}

La dernière partie de cet article sera consacrée à l'analyse des opportunités dans le cas du vol. Il s'agira de préciser la notion d'opportunité, d'en identifier les éléments et de montrer qu'elle 
peut nous aider à rendre compte du vol. Dans «Délinquants pourquoi?» cette notion était définie de la manière suivante : "Une opportunité naît quand un acteur peut, grâce à ses ressources, profiter des circonstances pour réaliser ses fins» (p. 168). L'analyse qui suivait portait sur l'éventail des opportunités légitimes et illégitimes qui jouent sur la décision d'un adolescent de s'engager dans une carrière criminelle. Avec le recul du temps, il faut reconnaître que la définition était trop englobante et que l'analyse ignorait la décision de commettre un délit pour se consacrer plutôt à la décision de s'engager dans une carrière criminelle. Or, pour un voleur, une opportunité c'est avant tout une bonne occasion de réaliser un vol lucratif. Et un voleur opportuniste sera celui qui sait profiter des circonstances, qui «saute» sur les occasions qui se présentent à lui. Dans le métro, un jeune homme qui a besoin d'argent voit que le sac à main de la dame qui lui tourne le dos est ouvert et laisse voir un porte monnaie bien garni. C'est en songeant à de telles situations que Hough, Clarke et Mayhew (1980) écrivaient : "Criminal opportunities exist not only where the material conditions are present but where benefits can be gained at low risk» (p. 5). L'opportunité peut alors être définie comme un contact entre un délinquant potentiel et une cible intéressante. L'opportunité apparaît quand sont réunies dans un lieu et à un moment donné les conditions favorables à la perpétration d'un crime. Si on part de cette idée, il est possible de formuler quelques propositions qui serviront à rendre compte d'un bon nombre d'observations concernant le choix des cibles, la victimisation, les décisions des délinquants, etc.

1. Les vols varient en raison directe des opportunités qui s'offrent aux voleurs potentiels.

2. L'opportunité est définie comme le contact entre un délinquant potentiel et une cible intéressante.

3. Le nombre de contacts entre les délinquants potentiels et les cibles intéressantes varie en raison directe du nombre de cibles et de leur accessibilité.

4. L'intérêt des cibles varie en raison directe de leur valeur et de leur vulnérabilité. Il varie en raison inverse de leur inertie.

Cinq notions seront explicitées à l'aide de quelques résultats de recherche :

1. Le nombre de cibles; 
2. L'accessibilité ;

3. La valeur ;

4. La vulnérabilité ;

5. L'inertie.

1. Le nombre de cibles. À première vue, il semblera évident que plus les cibles sont nombreuses, plus les délinquants potentiels auront d'opportunités et plus il y aura de vols. C'est oublier que la valeur d'un objet augmente avec sa rareté. Les objets rares risquent d'éveiller la convoitise. Le cas de l'automobile nous apportera quelques éléments de réponse. Wilkins (1964, p. 54) a rapporté qu'il y avait dans l'Angleterre de l'après-guerre un rapport très étroit entre le nombre de véhicules automobiles et les vols dans ces véhicules. Aux États-Unis, Gould (1969) a montré que, pour la période allant de 1950 à 1965, l'augmentation des véhicules automobiles allait de pair avec l'augmentation des vols d'automobile. Cependant, pendant les années 1930, l'augmentation des véhicules automobiles s'accompagna d'une baisse des vols de ces véhicules. L'interprétation de Gould repose sur le fait que, pendant cette période (la crise sévissait alors), les vols d'automobiles étaient commis par des professionnels. Or, plus les autos étaient rares, plus leur valeur de revente était grande. C'est ce qui encourageait les voleurs professionnels à pratiquer ce vol. On comprend alors pourquoi un plus grand nombre d'automobiles fait baisser leur vol : leur valeur de revente baisse. Selon Gould, en période d'abondance - en l'occurrence après la guerre de 1939 - la dynamique devient tout à fait différente. Les voleurs d'automobiles sont de plus en plus des jeunes et des amateurs. C'est l'âge d'or du «joy riding». Dans une telle situation, il y a un lien direct entre opportunitês et vols : plus les automobiles sont nombreuses, plus il y a d'opportunités et plus il y a de vols. Parallèlement joue le phénomène de la privation relative : quand la plupart des gens jouissent d'un bien, celui qui s'en trouve privé en conçoit un dépit plus grand et est fort tenté de se servir ${ }^{1}$.

1. Il est fort possible que nous retournions à une situation où le vol d'automobile soit de nouveau dominé par des professionnels. Il semble de plus en plus qu'on vole les automobiles pour les démonter et ensuite revendre les pièces. À l'époque du «joy riding», $90 \%$ des autos volées étaient retrouvées, maintenant on n'en retrouve plus que $53 \%$ (The Miami Herald 1/1/85). 
Si l'analyse de Gould est juste et si on peut la généraliser, il faudrait faire une importante réserve à la proposition selon laquelle le nombre de vols va de pair avec le nombre de cibles. Il faudrait alors la formuler ainsi : dans les sociétés d'abondance où le vol est dominé par des amateurs, plus les cibles sont nombreuses, plus il y aura de vols.

2. L'accessibilité des cibles. Plus les cibles sont accessibles aux délinquants potentiels, plus ceux-ci auront d'opportunités. Une cible est accessible quand le délinquant peut entrer en contact avec celle-ci avec le minimum de déplacements. Les deux composantes de l'accessibilité sont la proximité géographique et la proximité sociale.

A) La proximité géographique. De façon générale, les jeunes délinquants opèrent à faible distance de leur résidence. Dans une récente étude sur le cambriolage au Québec (Fréchette 1984, p. 18-19), les distances entre le domicile du cambrioleur et le lieu du délit s'échelonnaient comme suit :
- moins de $1 \mathrm{~km}: 11 \%$
- entre 1 et $2 \mathrm{~km}: 24 \%$
- entre 2 et $3 \mathrm{~km}: 11 \%$
- entre 3 et $4 \mathrm{~km}: 5 \%$
- entre 4 et $5 \mathrm{~km}: 3 \%$
- entre 5 et $9 \mathrm{~km}: 12 \%$

$(n=935)$

$51 \%$ des vols par effraction se produisent à moins de $4 \mathrm{~km}$ de la résidence du cambrioleur. De plus, $56 \%$ d'entre eux se déplaçaient à pied, contre $28 \%$ en voiture.

Les cambrioleurs aiment mieux opérer dans les quartiers qu'ils connaissent de première main, même s'ils sont habités par des gens pas très riches, que dans les quartiers qu'ils n'ont pas l'habitude de fréquenter (Reppetto, 1974). Ils se sentent plus en sécurité dans les secteurs qui leur sont familiers, où ils peuvent évoluer sans se faire remarquer et d'où ils peuvent fuir rapidement sans risquer d'être coincés dans un cul de $\operatorname{sac}^{2}$ (Maguire, 1982, p. 82). Le problème du transport ne peut être sous-estimé lui non plus. Les voleurs ne sont pas tous motorisés et ceux qui le sont préfèrent souvent ne pas prendre leur automobile.

2. On empiète ici sur la vulnérabilité. Voir un peu plus loin. 
Plusieurs autres résultats de recherche peuvent être analysés en termes d'accessibilité, comme en font foi les observations qui suivent.

Les délits sont souvent commis le long du trajet que prend le délinquant quand il va de sa résidence à son lieu de travail ou de loisir (Clarke et Cornish, 1985).

Les maisons les plus souvent cambriolées sont souvent à la limite entre un quartier riche et un quartier pauvre. Les cambrioleurs choisissent ces sites à cause de la facilité de fuite et aussi parce qu'ils se font moins remarquer dans de tels secteurs. (Brantingham et Brantingham, 1975.)

Les quartiers où il est facile de se retrouver (où, par exemple, les rues sont droites) ont un taux de victimisation plus élevé que les quartiers qui ont un plan compliqué (par exemple, les secteurs formés de rues en demi-cercle et de cul-de-sac). (Clarke et Cornish, 1985, p. 5.)

Les voleurs à main armée ont une certaine prédilection pour les endroits familiers. Ils s'en prennent à des banques qu'ils connaissent déjà, soit parce que c'est dans leur voisinage, soit parce qu'ils les ont déjà attaquées. "On s'attaque à la banque en face de chez soi, dans son quartier ou dans la région qu'on connaît bien» (Dionne, 1983, p. 123.)

B) La proximité sociale. Dans le cas des vols sur la personne, il peut arriver que la «cible» (il serait peut-être plus approprié de parler de la victime) aille vers le délinquant potentiel. Les adolescents sont trois fois plus souvent victimisés que les personnes âgées parce qu'ils sortent plus souvent et qu'ils ont un mode de vie qui les rapproche des délinquants potentiels (Hindelang, Gottfredson et Garofalo, 1978, Van Dijk et Steinmetz, 1980). En Angleterre, les personnes qui sortent fréquemment le soir et les fins de semaine courent un risque relativement élevé de victimisation (Gottfredson, 1984). Aux U.S.A., les travailleurs qui sont les plus souvent victimes de vols qualifiés et de vols simples se retrouvent dans les secteurs du loisir et de la restauration («Busboys», laveurs de vaisselle, waiter). La victimisation est aussi très fréquente dans les catégories d'emploi qui exigent des déplacements continuels : chauffeurs de taxi et livreurs de journaux (Block, Felson et Block, 1984). 
3. La valeur d'une cible est sa faculté de satisfaire le désir ou le besoin du délinquant potentiel'. C'est l'utilité au sens large de cette cible à ses yeux : sa rentabilité, sa valeur de revente, l'attirance qu'elle exerce, l'usage qu'il peut en faire. La dimension subjective de la notion de valeur mérite d'être soulignée. La valeur d'une cible dépendra des intérêts du voleur, de ses désirs, de ses besoins, de ses fins. Les fins que poursuit un voleur potentiel peuvent varier : l'expédient, la possession, l'utilité, la convoitise, le supplément, la fête... (Cusson, 1981, p. 112 à 118). La valeur monétaire des objets est évidemment importante mais, plus important, encore, sa valeur de revente. Une toile de maitre peut valoir très cher mais si le cambrioleur ne connaît personne qui soit prêt à la lui racheter, elle ne lui sera pas d'une grande valeur. Il n'est pas exclu que les fluctuations des prix sur le marché noir et sur le marché officiel aient une incidence sur les vols. On disait à Montréal que la hausse de l'or avait été suivie d'une recrudescence de vols de bijoux. Les "opérations identification» qui consistent à graver les objets qui risquent d'être volés contribuent à faire baisser leur valeur de revente. Or il semble qu'elles aient une certaine efficacité. Clarke (1983, p. 24) rapporte qu'elles peuvent faire baisser le niveau de cambriolage d'un secteur quand la majorité des familles de ce secteur participent à l'opération. L'importance des receleurs ne saurait être sous-estimée. Un des problèmes majeurs des cambrioleurs est de trotiver un receleur qui puisse lui acheter son butin à un bon prix (Maguire, 1982, p. 70).

4. La vulnérabilité peut se définir simplement par l'absence de risque pour le délinquant potentiel. Une cible est vulnérable quand le danger que l'on court en tentant de se l'approprier est relativement bas. Par définition, la vulnérabilité varie en raison inverse du risque d'être arrêté, d'être emprisonné, d'être blessé ou d'être tué.

Dans les hold-up, la vulnérabilité est un facteur de première importance au moment du choix de la cible. Sur 52 auteurs de vols à main armée du Québec interrogés, 31 disaient qu'ils tenaient compte en premier lieu des risques encourus alors que 21 considèrent d'abord l'argent disponible. L'un deux disait :

...c'est sûr y'a des places où y'a ben plus d'argent mais c'est impossible à faire. Faut aussi qu'tu penses à ta fuite. Vaut

3. Kuhlhorn et Svensson 1982 et Steinmetz (1982) préfèrent parler de l'attraction de la cible. 
mieux aller dans une place où y'a moins d'argent mais où t'as des bonnes chances de réussir... (Bellot, 1983, p. 35).

Les facteurs dont tiennent compte les cambrioleurs lors du choix de la cible et les informations qu'ils recherchent se rapportent souvent à la vulnérabilité. C'est ce que constate Reppetto (1974, p. 16 et 105).

Facteurs dont tiennent compte les cambrioleurs lors du choix de la cible

Un secteur dans lequel on peut passer inaperçu ................. 21\%

Maisons isolées ................................................................. 19\%

Absence de patrouille de police ........................................ 19\%

Les voisins ne se connaissent pas les uns les autres ........... $12 \%$

Informations que les cambrioleurs aimeraient obtenir avant d'agir

Les occupants sont-ils présents ? ................................... 70\%

Y a-t-il un système d'alarme ? .......................................... $36 \%$

Quelles sont les routes de fuite ? ..................................... 20\%

$\mathrm{Y}$ a-t-il des patrouilles de police ? .................................. 14\%

La vulnérabilité d'une cible est affectée par quatre facteurs principaux : 1) la présence de la victime, 2) la capacité défensive de la victime, 3) les difficultés de fuite, 4) la surveillance de tiers.

1. La présence de la victime potentielle ou de ses proches réduit souvent à néant la vuilnérabilité d'une oible. Nous avons vu l'importance de l'absence de gardien dans les recherches de Felson et de ses collègues. La présence ou l'absence des occupants à la maison est une considération primordiale pour les cambrioleurs (Reppetto, 1974, Maguire, 1982, Winchester et Jackson, 1983).

2. La capacité défensive de la victime potentielle. Les risques d'un voleur seront d'autant plus grands que la victime potentielle est résolue et capable de réagir en le dénonçant en l'arrêtant, en le blessant ou en le tuant. La liste qui suit donne une bonne idée des éléments qui peuvent contribuer à accroître la capacité défensive d'un individu :

- la force physique

- les chiens

- les armes

- la volonté de se défendre (les voleurs à main armée 
préfèrent souvent avoir affaire à un employé plutôt qu'au propriétaire qui est plus motivé à résister)

- les systèmes d'alarme et de détection.

3. Les difficultés de fuite. Tout ce qui empêche ou ralentit la fuite du voleur augmente ses risques. La fuite est une des principales préoccupations des voleurs à main armée. L'un deux disait : "Ce qui est dangereux, c'est pas de voler, c'est fuir, donc je planifie le get away» (Dionne, 1983, p. 123). Les cambrioleurs apprécient les résidences qui ont une porte arrière d'où ils peuvent fuir si les occupants arrivent par en avant. En Floride, un dépanneur est une bonne cible s'il est situé non loin d'une grande route mais pas directement sur cette route (Brantingham et Brantingham, 1934, p. 342). Au Québec, certains voleurs à main armée préfèrent les secteurs où la circulation automobile est à la fois dense et fluide ce qui permet une fuite à la fois discrète et rapide. (Bellot, 1983. p. 34). Parmi les autres circonstances qui peuvent faciliter la fuite, on peut mentionner les facilités de stationnement, la proximité d'une bouche de métro, l'obscurité...

4. La surveillance par des tiers. Le danger que court un délinquant potentiel est plus grand si la cible est surveillée par des tiers (employés, gardiens, policiers, voisins...). Encore faut-il que cette surveillance soit active, c'est-à-dire que les gens soient prêts à intervenir quand ils voient un individu aux allures louches. Les projets du type defensible space qui tablent beaucoup sur la surveillance "naturelle» et les programmes de surveillance de quartier n'ont pas donné beaucoup de résultats en partie parce que la motivation des simples citoyens à garder les yeux ouverts et, surtout, à intervenir n'est pas très forte (cf. Clarke, 1983 et Murray, 1983).

Le climat des villes modernes dissuade les gens à intervenir devant des agissements suspects. L'anonymat, l'indifférence à l'autre, l'encouragement à ne pas se mettre le nez dans les affaires des autres, tout cela augmente notre vulnérabilité au crime. Les gens ne veulent pas savoir ce qui se passe chez le voisin. De plus, ils ont tendance à donner une signification anodine à des gestes louches ou bizarres. Lors d'une expérience menée dans plusieurs villes américaines, le comparse d'un chercheur force la porte d'une automobile et y vole une caméra et d'autres objets. Presque tous les passants qui voient le vol passent leur chemin. Seulement $3 \%$ 
d'entre eux interviennent. Le fait que le voleur soit une femme ne change pas sensiblement les résultats (Steinmetz, 1982, p. 66).

Il a été démontré que la surveillance par un employé était un moyen efficace de prévenir le crime. Les blocs appartements qui ont un portier souffrent moins de cambriolage. Ceux qui ont un concierge souffrent moins de vandalisme. Dans les terrains de stationnement qui ont un employé, les vols d'autos sont moins nombreux. (Clarke, 1983, p. 240-1).

La simple possibilité d'être vu par les voisins peut dissuader certains voleurs. C'est ainsi que les cambriolages sont plus fréquents dans les maisons qui échappent à la surveillance des voisins, comme les maisons isolées et celles qui sont entourées d'un jardin et de buissons qui obstruent la vue (Clarke et Cornish, 1985).

5. L'inertie. Cette notion proposée par Felson mérite d'être retenue. On pourrait la définir comme la résistance qu'oppose un objet au déplacement ou à la pénétration. Plus l'inertie d'une cible est grande, moins le délinquant la trouvera intéressante. Le coffrefort fournit l'exemple parfait d'une cible qui a une grande inertie. Les variables suivantes peuvent contribuer à l'inertie d'une cible.

- le poids

- la dimension

- les serrures, cadenas et autres dispositifs qui réduisent la mobilité d'un objet pénétration

- les portes, les grilles et autres obstacles qui empêchent la

Les biens que les cambrioleurs au Québec recherchent tout particulièrement sont : l'argent $(25 \%)$, les appareils de son $(10 \%)$, l'alcool $(9 \%)$, de l'argent et des bijoux $(7 \%) \ldots$ (Fréchette, 1984, p. 18). En somme, des biens dont l'inertie est faible ou nulle.

Les mesures dites de «target hardening» ont une réelle efficacité préventive. En Allemagne fédérale, l'obligation d'installer des dispositifs antivol sur toutes les automobiles a fait baisser les vols d'auto de plus de $60 \%$. En Angleterre, les vols dans les téléphones publics ont été virtuellement éliminés en 
remplaçant le réceptacle à monnaie qui était en aluminium par un réceptacle en acier, beaucoup plus solide ${ }^{4}$. (Clarke, 1983, p. 24).

\section{CONCLUSION}

Plusieurs facettes du vol peuvent être appréhendées par la proposition qui dit que le vol suppose la rencontre entre un délinquant potentiel et une cible susceptible de le satisfaire au moindre risque. L'analyse des recherches sur le choix des cibles, sur les facteurs de victimisation et, de façon générale, sur les décisions des délinquants permet de donner un contenu assez spécifique à cette proposition. Celui-ci peut être saisi d'un seul coup d'œil dans le schéma qui suit.

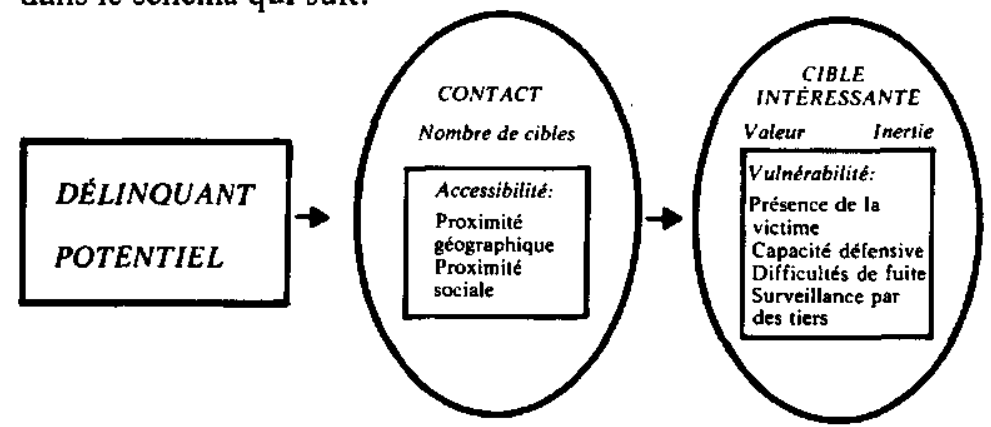

Il n'est peut-être pas sans intérêt de souligner que la notion de rationalité sous-tend l'essentiel du modèle. En effet, le schéma nous dit que le délinquant s'arrête sur la cible 1) qui a la plus grande valeur, 2) qu'il est peu risqué de voler, 3) qui est facile à déplacer ou à forcer et 4) qui est la plus accessible. Si on admet qu'une décision est rationnelle quand elle maximise les gains et minimise les coûts, celle du délinquant, telle qu'elle vient d'être présentée apparaît assez rationnelle. Il ne s'agit pas du tout de prétendre que la décision du voleur est la meilleure, mais plutôt de suggérer que, dans le cadre limité qui est le sien, le délinquant tend à l'efficacité.

Tout cela paraîtra peut-être bien trivial. Pourquoi s'abaisser à ces considérations si concrètes et si près du sens commun? Parce que notre but est de rendre compte des faits avec simplicité et

4. Il vaut la peine de préciser que les serrures, grilles, cadenas, etc., font plus qu'augmenter l'inertie de la cible; en outre, ils en réduisent la vulnérabilité. En effet, cela prend du temps de forcer une serrure ou un cadenas et cela fait du bruit. De ce fait, les risques du voleur augmentent. 
cohérence. Parce que notre souci est d'avoir prise sur la réalité. Parce que notre objectif est d'acquérir une perception organisée du monde visible.

Il serait ridicule de prétendre que l'analyse stratégique peut épuiser toute la question criminelle. Elle n'est qu'un point de vue parmi d'autres. Mais un point de vue qui mérite une place en criminologie. Tout simplement parce que nous apprenons quelque chose sur le crime quand nous le regardons sous l'angle de l'utilité pour son auteur. Depuis quelques années, la criminologie s'est éparpillée dans un foisonnement de recherches allant dans tous les azimuts. Il en est sorti un grand nombre d'observations intéressantes. Encore faut-il les classer et leur donner un sens autre que descriptif. L'analyse stratégique, sans avoir réponse à tout, peut nous aider à intégrer dans un modèle cohérent un bon nombre de faits dispersés.

\section{REFERENCES}

BELLOT, S. (1983), Portrait du voleur à main armée occasionnel, Montréal, Centre international de criminologie comparée, Université de Montréal, Rapport technique no 7.

BLOCK, R., M. FELSON, C. BLOCK (1984), Crime Victimization and the United States Occupational Siructure, Paper presented at the American Criminological Society, Cincinnati.

BRANTINGHAM, P.V., P.L. BRANTINGHAM (1975), .The Spacial Patterning of Burglary», Howard Journal of Penology and Crime Prevention, 14, p. 11-24. 
BRANTINGHAM, P., P.L. BRANTINGHAM (1978), A Theoretical Model of Crime Site Selection", in M.D. Krohn, R.L. Akers (édit.), Crime, Law and Sanctions, Beverly Hills, Calif., Sage

BRANTINGHAM, P., P.L. BRANTINGHAM (1984), Patterns in Crime. New York, Macmillan.

CLARKE, R.V.G. (1980), situationals Crime prevention: theory and practice», The British Journal of Criminology, 20, no 2, p. 136-147.

CLARKE, R.V.G. (1983), «Situational Crime Prevention: its theoretical Basis and Practical Scope», in M. Tonry, N. Morris, (édit.), Crime and Justice, no 4, p. 225-256.

CLARKE, R.V.G., D.B. CORNISH (1985), «Modelling Offender's decision: a framework for research and policys, in M. Tonry, N. Morris (édit.), Crime and Justice, vol. 6, Chicago, University of Chicago Press pp. 147-186.

CLARKE, R.V.G., P. MAYHEW (1980), Designing Out Crime, London, Her Majesty's stationary office.

COHEN, L.E., M. FELSON (1979), *Social change and crime rate trends: a routine activity approach», American Sociological Review, vol. 44, p. $588-608$.

COHEN, L.E., M. FELSON, K.C. LAND (1980), «Property crime rates in the United States: a macrodynamic analysis 1947-1977, with ex ante forecasts for the mid - 1980», American lournal of Sociology, 86, 90-118.

CUSSON, M. (1983), le Contrôle social du crime, Paris, Presses universitaires de France.

CUSSON, M. (1981), Délinquants pourquoi?, Montréal, Hurtubise, H.M.H.; Paris, Armand Colin.

DIJK, J.J.M., VAN, C.H.D. STEINMETZ (1980), The R.D.C: Victim Survey 1974-1979, The Hague, Research and Documentation Center.

DIONNE, M. (1984), Portrait de voleurs à main armée : les récidivistes et les professionnels, Montréal, Centre international de criminologie comparée, Université de Montréal, Rapport technique no 9.

FELSON, M. (1983), Ecology of Crimes, in Encyclopedia of Crime and Iustice, vol. 11, New York, The Free Press.

FELSON, M., L.E. COHEN (1980), «Human Ecology and Crime: A Routine Activity Approach», Human Ecology, vol. 8, p. 384-406.

FRECHETTE, P. (1984), «Le vol avec effraction: profil de son auteur», Sareté, novembre p. 11-22.

GOTTFREDSON, M.R. (1984), Victims of Crime: the Dimension of Risk, London, Home Office Research Studies Report, no 81 .

GOULD, L. (1969), The changing structure of property crime in an affluent society ", Social Forces, 48 : 50-59.

HINDELANG, M.J., C.S. DUSS, P. SUTTON, A.L. AUMICK (1977), Source book of Criminal Justice Statistics, 1976, Washington, D.C., U.S. Dept. of Justice L.E.A.A. U.S., Government Printing Office.

HINDELANG, M.J., M.R. GOTTFREDSON, J. GAROFALO (1978), Victims of Personal Crime: An Empirical Foundation for a Theory of Personal Victimization, Cambridge, Mass., Ballinger. 
HOUGH, J.M., R.V.G. CLARKE, P. MAYHEW (1980), eIntroduction ${ }_{\text {, }}$ in R.V.G. Clarke, P. Mayhew, Designing out Crime, London, Her Majesty's stationary office.

KUHLHORN, E., SVENSSON (1982), Crime Prevention, Stockholm, The National Swedish Council for Crime Prevention.

MAGUIRE, M. (1982), Burglary in a Dwelling, London, Heinemann.

MURRAY, C.A. (1983), The Physical Environment and Community Control of Crime, in J.Q. Wilson, (édit.), Crime and Public Policy, San Francisco, ICS Press.

REPPETTO, T.A. (1974), Residential Crime, Cambridge, Mass., Ballinger STEINMETZ, C.H. (1982), *A first step toward victimological risk analysis», in E. Kuhlhorn, B. Svensson, Crime Prevention, Stockholm, The National Swedish Council for crime prevention.

WILKINS, L. (1964), Social Deviance, Englewood Cliff, N.J., Prentice-Hall; London, Tavistock.

WINCHESTER, S., H. JACKSON (1982), Residential Burglary: The limits of Prevention, London, H.M.S.O., Home Office Research Study, no 74. 